\title{
Variation of the $O s G I$ intron and its phenotypic associations in Oryza rufipogon Griff. and Oryza sativa L.
}

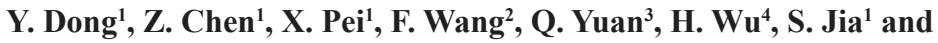 \\ Y. Peng ${ }^{4}$ \\ ${ }^{1}$ Institute of Biotechnology, Chinese Academy of Agricultural Sciences, \\ Beijing, China \\ ${ }^{2}$ Rice Research Institute, Guangdong Academy of Agricultural Sciences, \\ Guangzhou, China \\ ${ }^{3}$ MOE Key Lab of Tropical Biological Resources, \\ College of Agriculture Science, Hainan University, Haikou, China \\ ${ }^{4}$ Institute of Plant Protection, Chinese Academy of Agricultural Sciences, \\ Beijing, China
}

Corresponding authors: Y. Peng / X. Pei

E-mail: pyf@caascose.net.cn / peixw@mail.caas.net.cn

Genet. Mol. Res. 12 (3): 2652-2669 (2013)

Received November 30, 2012

Accepted March 20, 2013

Published July 30, 2013

DOI http://dx.doi.org/10.4238/2013.July.30.4

ABSTRACT. We analyzed intron 9 of the Os GI gene in Oryza rufipogon and Oryza sativa in order to investigate evolutionary relationships in rice and the relationship between intron variation and phenotype. OsGI-9 was cloned in 38 O. rufipogon populations and in 139 O. sativa cultivars and the phylogeny was reconstructed. Seed cold tolerance and dormancy were quantified in $O$. sativa. Three $O s G I-9$ band types occurred in O. rufipogon: S-type $(1.2 \mathrm{~kb})$, F-type $(0.9 \mathrm{~kb})$, and FS-type $(1.2$ and $0.9 \mathrm{~kb})$, whereas only the S-type and F-type occurred in $O$. sativa. The S-type contains two 255-bp repeats, the F-type contains one 255-bp repeat, and the FS-type contains both. All individuals could be divided into 5 groups in the organism's phylogenetic network: S-type 
$O$. rufipogon, F-type $O$. rufipogon, FS-type $O$. rufipogon, S-type $O$. sativa, and F-type $O$. sativa. F-type $O$. sativa are most closely related to F-type $O$. rufipogon and S-type $O$. sativa are most closely related to S-type O. rufipogon. Statistical analysis indicated that OsGI-9 type is significantly correlated with phenotype; most S-type $O$. sativa have strong seed dormancy and cold tolerance, and most F-type $O$. sativa have no seed dormancy and poor cold tolerance.

Key words: Oryza rufipogon; Oryza sativa; OsGI; Phylogenetic network; Cold tolerance; Seed dormancy

\section{INTRODUCTION}

Oryza rufipogon is commonly recognized as the progenitor of Oryza sativa and is widely distributed in China, Southeast Asia and Southern Asia. Although both species reproduce asexually and sexually, asexual reproduction predominates in O. rufipogon. A high rate of cross-pollination, low reproductive isolation between $O$. rufipogon and $O$. sativa, and rich genetic diversity are valuable traits that have contributed to the improvement of $O$. sativa varieties (Lu et al., 2002). Although useful variations in $O$. sativa can be rapidly identified using genomic techniques and have been widely exploited via transgenic technology (Rakshit et al., 2007), approximately $30-40 \%$ of genetic variation was lost during rice domestication. Therefore, a valuable source of genetic variation remains within the wild germplasm (Sun et al., 2001), and it is therefore extremely important to characterize the genetic diversity of $O$. rufipogon and its phylogenetic relationship with $O$. sativa.

Phylogenetic analysis plays an important role in the characterization of evolutionary relationships between species and in the reconstruction of evolutionary processes (Lessa, 1992). Therefore, construction of reliable phylogenetic trees, using appropriate molecular markers and making full use of the phylogenetic information contained therein, is vital (Slade et al., 1994). Previous studies have used morphological features, data from cell research, and molecular markers such as isozymes or RFLP, RAPD, AFLP, SSR, SINEs, and MITE for phylogenetic analyses of A-type genome species (Ren et al., 2003). However, the species' classifications are currently unclear due to the lack of obvious morphological differences and because evolutionary relationships described in different studies are not consistent (Ren et al., 2003). Investigations into the huge potential of nuclear genes for phylogenetic analysis have increased in recent years (Slade et al., 1994; Sang, 2002). However, phylogenetic reconstruction at lower taxonomic levels has been limited due to a lack of sufficient variation within molecular sequences (Doyle et al., 1996). Intron sequences have been widely applied for the reconstruction of phylogeny at lower classification levels as they are rapidly evolving, easily cloned and nearly neutral (Dalebout et al., 2008). Nuclear genes, including introns, in diploid organisms can be homozygous or heterozygous, as a result of insertions or deletions (InDel) or base sequence variation (Creer et al., 2007). Increasing numbers of studies have indicated that hybrid introns, termed intra-individual allele heterozygotes (IIAHs), are common in nature. IIAHs provide a rich source of phylogenetic information, and can reveal potential hybridization or introgression in the analysis of phylogenetic relationships between related species or genetic structures within a species. 
Haplotypes are examined in the majority of current phylogenetic analyses; however, usually only a single IIAH haplotype is examined, and the phylogenetic information contained in hybrid introns has been so far neglected. In addition, increasing numbers of studies have indicated that intron diversity is related to many human diseases and phenotypic variations in animals and plants (Reszka et al., 2006). High outcrossing rates and intron heterozygosity are ubiquitous in O. rufipogon (Lu et al., 2002). Therefore, phylogenetic information contained in IIAHs should not be ignored during the reconstruction of $O$. rufipogon phylogeny using introns and for analyses of intron function.

Gigantea $(G I)$ is a circadian clock-controlled gene that regulates photoperiodic flowering in Arabidopsis (Fowler et al., 1999), and also plays a role in phytochrome B signaling, the circadian clock, carbohydrate metabolism, fruit setting, and cold stress response (Brock et al., 2007). OsGI, a single-copy gene, was identified as the rice ortholog of Arabidopsis GI and is an important regulator of flowering. In this study, the $O s G I$ intron in 38 Chinese O. rufipogon samples and in $139 \mathrm{O}$. sativa cultivars from around the world were used for phylogenetic reconstruction, and the correlation between $O s G I$ intron variation and $O$. sativa phenotypes were analyzed in order to determine the impact of $O s G I$ intron variation on rice evolution.

\section{MATERIAL AND METHODS}

\section{Material collection}

Thirty-eight $O$. rufipogon populations from China were selected for this study. Twelve populations were obtained from the Hainan Island Province and 26 populations were obtained from 6 inland Provinces: Guangdong $(\mathrm{N}=5)$, Guangxi $(\mathrm{N}=17)$, Yunnan $(\mathrm{N}=1)$, Hunan $(\mathrm{N}$ $=1)$, Fujian $(\mathrm{N}=1)$, Jiangxi $(\mathrm{N}=1)$ (Figure 1). The leaves of individual plants were collected from the populations in Hainan Island, Guangdong, Jiangxi, and Yunnan Provinces. To avoid replication of the same clones, samples were collected at intervals greater than $12 \mathrm{~m}$ (Xie et al., 2001). O. rufipogon seeds were collected from the populations in Guangxi, Hunan, and Fujian Provinces. Detailed sampling information for all of the $O$. rufipogon samples is provided in Table 1. The O. rufipogon samples from Hainan Island, Guangdong, Yunnan, and Jiangxi Provinces were collected by members of our laboratory, and the other samples were provided by the Crop Science Research Institute and the China National Rice Research Institute at the Chinese Academy of Agricultural Sciences and the Guangdong Academy of Agricultural Sciences. The leaves of individual plants from each population were clipped for DNA extraction. Seed samples were germinated on Petri dishes using moist filter paper and the seedlings were harvested for DNA extraction.

The seeds of 139 O. sativa cultivars were collected from around the world; 65 cultivars were from various provinces in China and 74 cultivars were from other countries (Table 1). All of the seeds were germinated and the seedlings were harvested for DNA extraction.

\section{Gene amplification and sequencing strategy}

Nuclear alleles within $O$. rufipogon individuals may be heterozygous due to the high cross-pollination rate; therefore, it was necessary to account for allele variation. In order to find individual allelic differences and avoid errors during polymerase chain reaction (PCR) 
amplification, genomic DNA was extracted from each individual using a high-fidelity polymerase (AccuPrime Taq DNA polymerase, Invitrogen) for PCR amplification, and at least 7 clones from each individual were sequenced. Mutations between haplotypes of a single individual located at informative sites were considered to be allelic variation, while mutations located at singleton variable sites were considered to be errors from PCR amplification.

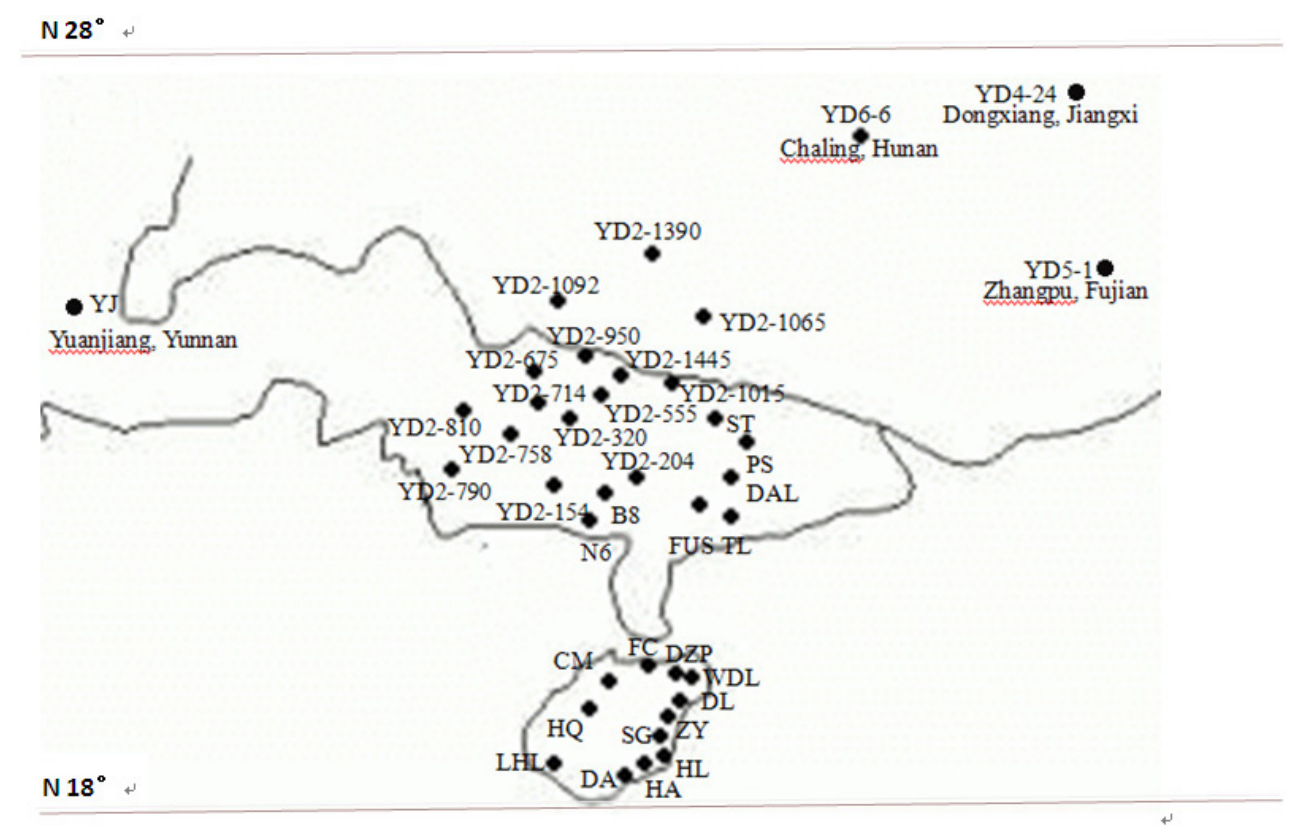

Figure 1. Distribution map of Oryza rufipogon populations from China.

\section{DNA extraction, PCR amplification, cloning, and sequence analysis}

Based on the sequence of $O s G I$ from $O$. sativa japonica Nipponbare (GenBank accession AP003047), specific primers were designed for $O s G I$ intron 9 (OsGI-9) using the exonprimed, intron-crossing method (forward 5'-ACAATGGCAAGTATAGGCTCC-3' and reverse 5'-ATCTTCATTTCCTGCGTGC-3'; Figure 2). Total DNA was extracted using a modification of the CTAB method (Xie et al., 1999). PCR amplification was performed in a total volume of $50 \mu \mathrm{L}$, containing $1 \mathrm{X}$ PCR buffer, $2.5 \mathrm{mM} \mathrm{MgCl}_{2}, 0.2 \mathrm{mM}$ dNTPs, $0.5 \mu \mathrm{M}$ of each primer, $0.025 \mathrm{U} / \mu \mathrm{L}$ AccuPrime Taq DNA (Invitrogen), and $5 \mathrm{ng} / \mu \mathrm{L}$ template DNA. Amplification was carried out for $5 \mathrm{~min}$ at $94^{\circ} \mathrm{C}$ followed by 35 cycles of $30 \mathrm{~s}$ at $94^{\circ} \mathrm{C}, 30 \mathrm{~s}$ at $54^{\circ} \mathrm{C}$, and $80 \mathrm{~s}$ at $72^{\circ} \mathrm{C}$, with a final extension at $72^{\circ} \mathrm{C}$ for $10 \mathrm{~min}$. The amplification products were separated by electrophoresis on $1.2 \%$ agarose gels, stained with ethidium bromide and images were captured using a gel imaging system (GBox, Gene Company). PCR fragments were purified using a DNA gel extraction kit (Axygen Biosciences) and cloned into the pEASY-T3 cloning vector (Transgen Biotech). Independent plasmids were randomly selected and at least 7 positive clones were individually sequenced, aligned and analyzed using Vector NTI advance 10 (Invitrogen). 
Table 1. Detailed information on the Oryza rufipogon and $O$. sativa samples used in this study.

\begin{tabular}{|c|c|c|c|c|}
\hline Code & Origin & Taxa & Samples type & Number of samples \\
\hline$\overline{\mathrm{DA}}$ & Dong'ao, Hainan Province, China & O. rufipogon & Individual plant & 128 \\
\hline DZP & Dazhipo, Hainan Province, China & O. rufipogon & Individual plant & 18 \\
\hline LHL & Ledong, Hainan Province, China & O. rufipogon & Individual plant & 7 \\
\hline SG & Shangen, Hainan Province, China & O. rufipogon & Individual plant & 3 \\
\hline HQ & Danzhou, Hainan Province, China & O. rufipogon & Individual plant & 14 \\
\hline WDL & Donglu, Hainan Province, China & O. rufipogon & Individual plant & 14 \\
\hline ZY & Zhongyuan, Hainan Province, China & O. rufipogon & Individual plant & 13 \\
\hline DL & Dalu, Hainan Province, China & O. rufipogon & Individual plant & 16 \\
\hline HA & Houan, Hainan Province, China & O. rufipogon & Individual plant & 4 \\
\hline HL & Hele, Hainan Province, China & O. rufipogon & Individual plant & 3 \\
\hline $\mathrm{FC}$ & Fuchen, Hainan Province, China & O. rufipogon & Individual plant & 4 \\
\hline $\mathrm{CM}$ & Chengmai, Hainan Province, China & O. rufipogon & Individual plant & 7 \\
\hline YJ & Yuanjiang, Yunnan Province, China & O. rufipogon & Individual plant & 18 \\
\hline PS & Pengshan, Guangdong Province, China & O. rufipogon & Individual plant & 16 \\
\hline ST & Shatian, Guangdong Province, China & O. rufipogon & Individual plant & 15 \\
\hline $\mathrm{TL}$ & Tanlu, Guangdong Province, China & O. rufipogon & Individual plant & 17 \\
\hline DAL & Daling, Guangdong Province, China & O. rufipogon & Individual plant & 13 \\
\hline FUS & Fushi, Guangdong Province, China & O. rufipogon & Individual plant & 6 \\
\hline YD4-24 & Dongxiang, Jiangxi Province, China & O. rufipogon & Individual plant & 52 \\
\hline N6 & Beihai, Guangxi Province, China & O. rufipogon & Seeds & 10 \\
\hline B8 & Bobai, Guangxi Province, China & O. rufipogon & Seeds & 10 \\
\hline YD2-154 & Lingshan, Guangxi Province, China & O. rufipogon & Seeds & 10 \\
\hline YD2-204 & Yulin, Guangxi Province, China & O. rufipogon & Seeds & 10 \\
\hline YD2-320 & Guixian, Guangxi Province, China & O. rufipogon & Seeds & 10 \\
\hline YD2-555 & Guiping, Guangxi Province, China & O. rufipogon & Seeds & 10 \\
\hline YD2-675 & Wuming, Guangxi Province, China & O. rufipogon & Seeds & 10 \\
\hline YD2-714 & Hengxian, Guangxi Province, China & O. rufipogon & Seeds & 10 \\
\hline YD2-758 & Fusui, Guangxi Province, China & O. rufipogon & Seeds & 10 \\
\hline YD2-790 & Zongzuo, Guangxi Province, China & O. rufipogon & Seeds & 10 \\
\hline YD2-810 & Long'an, Guangxi Province, China & O. rufipogon & Seeds & 10 \\
\hline YD2-950 & Shanglin, Guangxi Province, China & O. rufipogon & Seeds & 10 \\
\hline YD2-1015 & Tengxian, Guangxi Province, China & O. rufipogon & Seeds & 10 \\
\hline YD2-1065 & Hexian, Guangxi Province, China & O. rufipogon & Seeds & 10 \\
\hline YD2-1092 & Liucheng, Guangxi Province, China & O. rufipogon & Seeds & 10 \\
\hline YD2-1390 & Xiangshan, Guangxi Province, China & O. rufipogon & Seeds & 10 \\
\hline YD2-1445 & Wuxuan, Guangxi Province, China & O. rufipogon & Seeds & 10 \\
\hline YD5-1 & Zhangpu, Fujian Province, China & O. rufipogon & Seeds & 10 \\
\hline YD6-66 & Chaling, Hunan Province, China & O. rufipogon & Seeds & 10 \\
\hline Mudanjiang 19 & Heilongjiang Province, China & O. sativa & Seeds & 10 \\
\hline Ji $91-2605$ & Jilin Province, China & O. sativa & Seeds & 10 \\
\hline Liao 201 & Liaoning Province, China & O. sativa & Seeds & 10 \\
\hline $86 \times W-17$ & Ningxia Province, China & O. sativa & Seeds & 10 \\
\hline 9011 & Xinjiang Province, China & O. sativa & Seeds & 10 \\
\hline $91-13-11$ & Shangxi Province, China & O. sativa & Seeds & 10 \\
\hline Laolongxu & Shangxi Province, China & O. sativa & Seeds & 10 \\
\hline Xinong 8116 & Shangxi Province, China & O. sativa & Seeds & 10 \\
\hline Qiannong 5782 & Guizhou Province, China & O. sativa & Seeds & 10 \\
\hline Jinxi 870441 & Shanxi Province, China & $O$. sativa & Seeds & 10 \\
\hline Kenyu 16 & Hebei Province, China & O. sativa & Seeds & 10 \\
\hline Zhonghua 11 & Beijing, China & $O$. sativa & Seeds & 10 \\
\hline Zhonghua 8 & Beijing, China & $O$. sativa & Seeds & 10 \\
\hline Zhongzuo 8604 & Beijing, China & O. sativa & Seeds & 10 \\
\hline 6017 & Beijing, China & O. sativa & Seeds & 10 \\
\hline Yinfang & Tianjing, China & O. sativa & Seeds & 10 \\
\hline Putaohuang & Tianjing, China & O. sativa & Seeds & 10 \\
\hline Lujing 1 & Shandong Province, China & O. sativa & Seeds & 10 \\
\hline Zhengdao 5 & Henan Province, China & O. sativa & Seeds & 10 \\
\hline Xinyang 14 & Henan Province, China & O. sativa & Seeds & 10 \\
\hline Wanjing 1 & Anhui Province, China & O. sativa & Seeds & 10 \\
\hline Anxuan 4 & Anhui Province, China & O. sativa & Seeds & 10 \\
\hline Liushizao & Anhui Province, China & O. sativa & Seeds & 10 \\
\hline Wuyujing & Jiangsu Province, China & O. sativa & Seeds & 10 \\
\hline Shuangqing & Jiangsu Province, China & O. sativa & Seeds & 10 \\
\hline Nanjing 11 & Jiangsu Province, China & O. sativa & Seeds & 10 \\
\hline
\end{tabular}

Continued on next page 


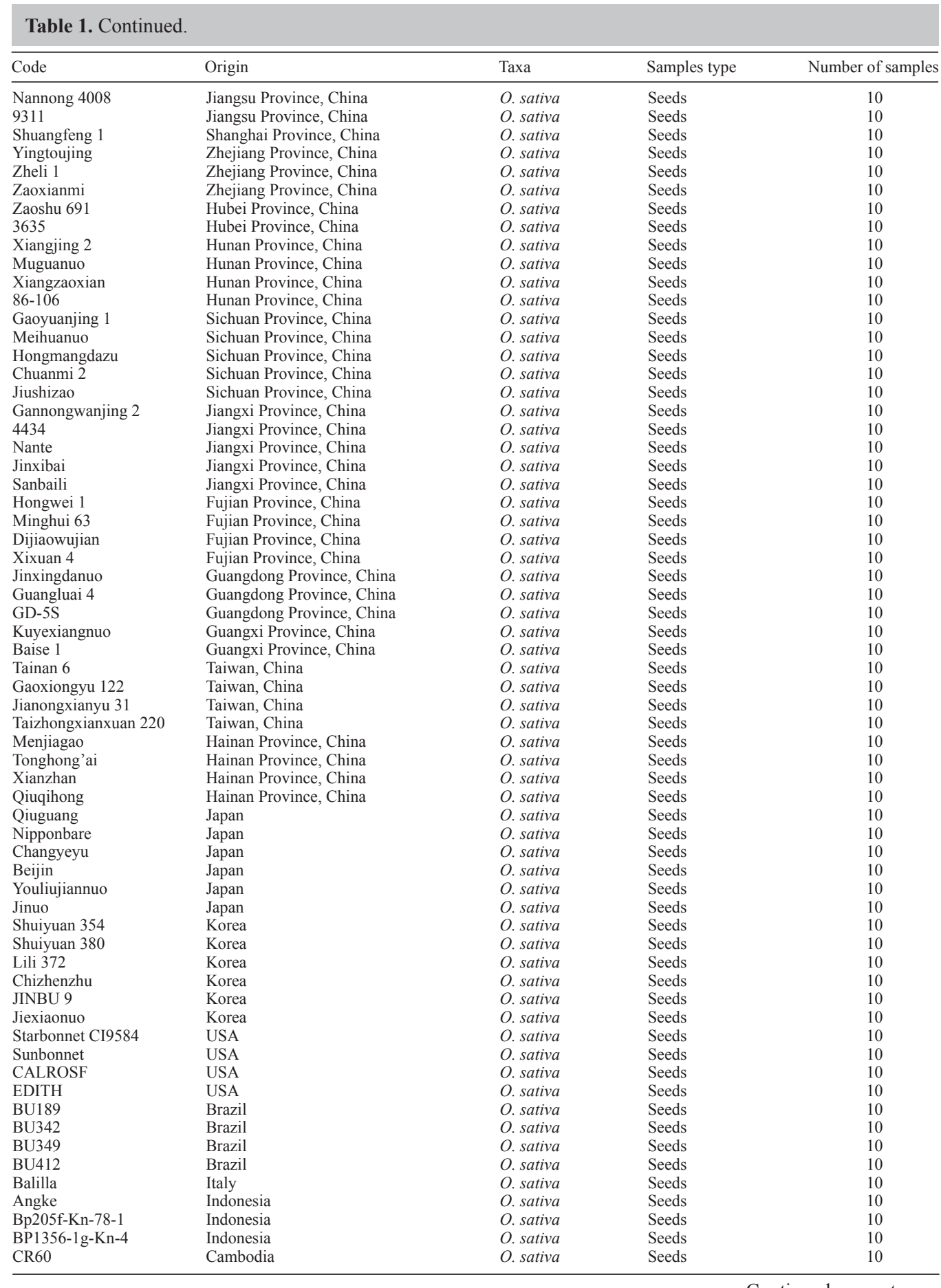

Continued on next page 


\begin{tabular}{|c|c|c|c|c|}
\hline Code & Origin & Taxa & Samples type & Number of samples \\
\hline $1 v-139$ & Vietnam & O. sativa & Seeds & 10 \\
\hline NR11 & Vietnam & $O$. sativa & Seeds & 10 \\
\hline VR345 & Vietnam & O. sativa & Seeds & 10 \\
\hline VR349 & Vietnam & O. sativa & Seeds & 10 \\
\hline VR350 & Vietnam & O. sativa & Seeds & 10 \\
\hline VR340 & Vietnam & O. sativa & Seeds & 10 \\
\hline VR347 & Vietnam & O. sativa & Seeds & 10 \\
\hline Matant MF & India & O. sativa & Seeds & 10 \\
\hline Mulant of dwarf & India & O. sativa & Seeds & 10 \\
\hline PSRM1-17 & India & O. sativa & Seeds & 10 \\
\hline RP1667-301-1196-1562 & India & O. sativa & Seeds & 10 \\
\hline RP1670-1418-2205-1582 & India & $O$. sativa & Seeds & 10 \\
\hline Toga & India & O. sativa & Seeds & 10 \\
\hline CNTLR85033-9-3-1-1 & Thailand & O. sativa & Seeds & 10 \\
\hline SPR85163-5-1-2 & Thailand & O. sativa & Seeds & 10 \\
\hline C. Costo & Burma & O. sativa & Seeds & 10 \\
\hline Mya-1 & Burma & O. sativa & Seeds & 10 \\
\hline Mya-2 & Burma & O. sativa & Seeds & 10 \\
\hline Manan Thukho & Burma & O. sativa & Seeds & 10 \\
\hline FAON11 & Burma & O. sativa & Seeds & 10 \\
\hline AZUCENA & Philippines & O. sativa & Seeds & 10 \\
\hline IR67406-6-3-2-3 & IRRI & O. sativa & Seeds & 10 \\
\hline IR70416-53-2-2 & IRRI & O. sativa & Seeds & 10 \\
\hline IR70445-146-3-3 & IRRI & O. sativa & Seeds & 10 \\
\hline $\mathrm{Bg} 300$ & Sri Lanka & O. sativa & Seeds & 10 \\
\hline Bg304 & Sri Lanka & O. sativa & Seeds & 10 \\
\hline $\mathrm{Bg} 305$ & Sri Lanka & O. sativa & Seeds & 10 \\
\hline Bg358 & Sri Lanka & $O$. sativa & Seeds & 10 \\
\hline Bg359 & Sri Lanka & O. sativa & Seeds & 10 \\
\hline NO.1 & Madagascar & O. sativa & Seeds & 10 \\
\hline HNR-2 & Madagascar & O. sativa & Seeds & 10 \\
\hline HNR-5 & Madagascar & O. sativa & Seeds & 10 \\
\hline HNR-17 & Madagascar & O. sativa & Seeds & 10 \\
\hline Khao Toum & Laos & O. sativa & Seeds & 10 \\
\hline Mack Kouk & Laos & O. sativa & Seeds & 10 \\
\hline SLK 2-21-4 & Laos & O. sativa & Seeds & 10 \\
\hline Mollika & Nepal & O. sativa & Seeds & 10 \\
\hline NR10073-167-3-1-1 & Nepal & $O$. sativa & Seeds & 10 \\
\hline NR10068-60-5-2 & Nepal & O. sativa & Seeds & 10 \\
\hline NR10078-76-1-1 & Nepal & O. sativa & Seeds & 10 \\
\hline Aus257 & Bengal & O. sativa & Seeds & 10 \\
\hline BR061-2B-25 & Bengal & O. sativa & Seeds & 10 \\
\hline BR319-1-HR28 & Bengal & O. sativa & Seeds & 10 \\
\hline UGEY MAP & Bhutan & O. sativa & Seeds & 10 \\
\hline 80A86YR72 & Australia & O. sativa & Seeds & 10 \\
\hline 80A90YR73 & Australia & O. sativa & Seeds & 10 \\
\hline 80A97YR74 & Australia & O. sativa & Seeds & 10 \\
\hline 80A97YR30 & Australia & O. sativa & Seeds & 10 \\
\hline 71011 & Australia & O. sativa & Seeds & 10 \\
\hline
\end{tabular}

The materials from Hainan and Guangdong, China, were collected by our laboratory. The other materials were provided by the Crop Science Research Institute and China Rice Research Institute at the Chinese Academy of Agricultural Science or the Academy of Agricultural Science in Guangdong Province.

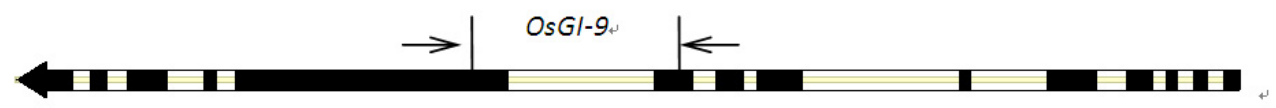

exon

intron

Figure 2. Scheme of the $O s G I$ gene. $O s G I-9$ was the amplified region in the study. 


\section{Phylogenetic analysis of heterozygous alleles}

As both $O$. rufipogon and $O$. sativa are diploid plants, O. rufipogon and $O$. sativa phylogeny were analyzed in the following manner. The haplotypes were first aligned using ClustalX 2.0 and exported in PHYLIP format (Larkin et al., 2007). The haplotype alignment was then transformed into a haplotype distance matrix using DNADIST in the PHYLIP 3.69 software (Felsenstein, 2008), transformed into an individual distance matrix by the host-associate method using the PBC software (Göker and Grimm, 2008), and the phylogenetic organism network was reconstructed by the NeighborNet method using the Splitstree4.0 software (Bryant and Moulton, 2004; Huson and Bryant, 2006).

\section{Seed dormancy and cold tolerance evaluation}

All of the $O$. sativa seeds used in this study were stored at $4{ }^{\circ} \mathrm{C}$ to maintain dormancy (Wang et al., 2009), and 100 seeds of each cultivar were selected to determine the germination rate (GR) and germination rate index (GRI). GR is the percentage of germinated seeds and GRI reflects the strength of seed germination. The seeds were soaked in $75 \%$ alcohol for $10 \mathrm{~min}$, washed with sterile distilled water and arranged on 9-cm Petri dishes covered with 2 layers of wet filter paper. The seeds were divided into 2 groups; one group was cultured at $14^{\circ} \mathrm{C}$ and the other at $30^{\circ} \mathrm{C}$. Both groups were kept moist with a 12-h light/12-h dark cycle and GR and GRI were determined after 25 days of culture. Seed dormancy was divided into 5 levels according to the $\mathrm{GR}$ at $30^{\circ} \mathrm{C}$ : GR $>80 \%$, non-dormant; 50 to $\leq 80 \%$, mild dormancy; 30 to $\leq 50 \%$, moderate dormancy; 5 to $\leq 30 \%$, strong dormancy; $\leq 5 \%$, very strong dormancy. Seed cold tolerance was estimated according to the difference between GR and GRI at $14^{\circ}$ and $30^{\circ} \mathrm{C}$ (Cao et al., 2001; Miura et al., 2004; Wang et al., 2009) as follows:

$$
\begin{aligned}
& \text { GR }(\%)=(\text { number of generated seeds } / \text { total number of seeds }) \times 100 \\
& \mathrm{GRI}=\mathrm{G}_{1} / \mathrm{T}_{1}+\mathrm{G}_{2} / \mathrm{T}_{2}+\ldots+\mathrm{G}_{\mathrm{n}-1} / \mathrm{T}_{\mathrm{n}-1}+\mathrm{G}_{\mathrm{n}} / \mathrm{T}_{\mathrm{n}}
\end{aligned}
$$

where $\mathrm{G}_{1}$ is the number of germinated seeds on day $T_{l}, G_{n}$ is the number of germinated seeds on day $T_{n} ; T_{1}$ is the day number at the first count, and $T_{n}$ is the day number at the last count.

\section{Statistical analysis}

Data were analyzed using SPSS 13.0 (Chicago, IL, USA) using Spearman's correlation and Kendall's method of non-parametric correlations for correlation analyses, and MannWhitney non-parametric tests.

\section{RESULTS}

\section{OsGI-9 in O. rufipogon and O. sativa}

The $O s G I-9$ regions in 516 individuals from 38 populations were amplified using PCR. Electrophoresis indicated the presence of 3 different band types in O. rufipogon: S-type (1.2 kb), F-type (0.9 kb), and FS-type (1.2 and $0.9 \mathrm{~kb}$; Figure 3). FS-type individuals were 
present in 18 O. rufipogon populations, including 7 populations from Hainan Island and 11 populations from inland China. S-type individuals were present in 19 O. rufipogon populations, including 4 populations from Hainan Island and 15 populations from inland China. F-type individuals were present in 9 O. rufipogon populations, including 5 populations from Hainan Island and 4 populations from inland China (Table 2).

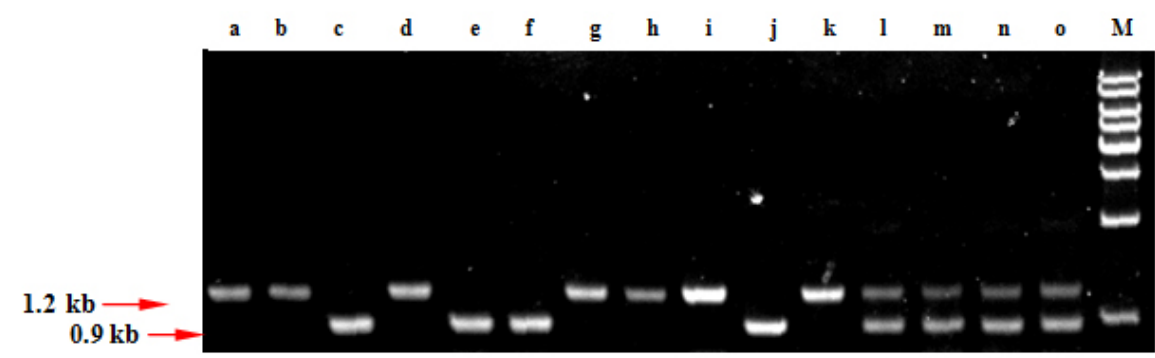

Figure 3. Band types of OsGI-9 in Oryza rufipogon individuals from the DZP population. Lanes $a-o=$ different individuals in the DZP population. Lanes $a, b, d, g, h, i, k=1.2 \mathrm{~kb}$; lanes $c, e, f, j=0.9 \mathrm{~kb}$; lanes $l, m, n, o=0.9$ and 1.2 $\mathrm{kb}$; lane $M=$ molecular marker.

Table 2. OsGI-9 band types in Oryza rufipogon.

\begin{tabular}{|c|c|c|c|}
\hline \multirow[t]{2}{*}{ Code } & \multicolumn{3}{|c|}{ OsGI-9 band types } \\
\hline & $\mathrm{F}(0.9 \mathrm{~kb})$ & $\mathrm{S}(1.2 \mathrm{~kb})$ & $\mathrm{FS}(0.9$ and $1.2 \mathrm{~kb})$ \\
\hline$\overline{\mathrm{DZP}}$ & $4 / 18^{*}$ & $10 / 18$ & $4 / 18$ \\
\hline LHL & - & $4 / 7$ & $3 / 7$ \\
\hline $\mathrm{SG}$ & - & - & $3 / 3$ \\
\hline $\mathrm{HQ}$ & - & - & $14 / 14$ \\
\hline WDL & - & - & $14 / 14$ \\
\hline $\mathrm{ZY}$ & $3 / 13$ & - & $10 / 13$ \\
\hline DL & & - & $16 / 16$ \\
\hline $\mathrm{HA}$ & $4 / 4$ & - & - \\
\hline $\mathrm{HL}$ & $3 / 3$ & - & - \\
\hline $\mathrm{FC}$ & - & $4 / 4$ & - \\
\hline $\mathrm{CM}$ & - & $7 / 7$ & - \\
\hline DA & $128 / 128$ & - & - \\
\hline $\mathrm{YJ}$ & - & $18 / 18$ & \\
\hline PS & - & $13 / 16$ & $3 / 16$ \\
\hline ST & $15 / 15$ & - & - \\
\hline TL & $3 / 17$ & $2 / 17$ & $12 / 17$ \\
\hline DAL & - & - & $13 / 13$ \\
\hline FUS & & $4 / 6$ & $2 / 6$ \\
\hline $\mathrm{YD}_{4}-24$ & - & $52 / 52$ & - \\
\hline $\mathrm{N} 6{ }^{4}$ & - & $10 / 10$ & - \\
\hline B8 & & $10 / 10$ & - \\
\hline $\mathrm{YD}_{2}-154$ & $10 / 10$ & - & - \\
\hline $\mathrm{YD}_{2}^{2}-204$ & - & - & $10 / 10$ \\
\hline $\mathrm{YD}_{2}^{2}-320$ & - & $10 / 10$ & - \\
\hline $\mathrm{YD}_{2}^{2}-555$ & - & - & $10 / 10$ \\
\hline $\mathrm{YD}_{2}^{2}-675$ & - & $10 / 10$ & \\
\hline $\mathrm{YD}_{2}^{2}-714$ & - & - & $10 / 10$ \\
\hline $\mathrm{YD}_{2}^{2}-758$ & - & - & $10 / 10$ \\
\hline $\mathrm{YD}_{2}^{2}-790$ & - & $10 / 10$ & \\
\hline $\mathrm{YD}_{2}^{2}-810$ & - & & $10 / 10$ \\
\hline $\mathrm{YD}_{2}^{2}-950$ & - & $10 / 10$ & \\
\hline $\mathrm{YD}_{2}^{2}-1015$ & - & - & $10 / 10$ \\
\hline $\mathrm{YD}_{2}^{2}-1065$ & - & $10 / 10$ & - \\
\hline $\mathrm{YD}_{2}^{2}-1092$ & - & $10 / 10$ & \\
\hline $\mathrm{YD}_{2}^{2}-1390$ & - & & $10 / 10$ \\
\hline $\mathrm{YD}_{2}^{2}-1445$ & - & $10 / 10$ & - \\
\hline $\mathrm{YD}_{5}^{2}-1$ & $10 / 10$ & & \\
\hline $\mathrm{YD}_{6}^{5}-66$ & . & $10 / 10$ & - \\
\hline
\end{tabular}

*Means that 4 individuals among 18 samples from DZP have F-type of OsGI-9. 
S-type and FS-type $O$. rufipogon individuals were predominant in the inland region of China, with a slightly larger number of S-type individuals. O. rufipogon individuals from Yuanjiang, Yunnan Province (YJ), which are thought to be a typical representation of $O$. rufipogon (Sun et al., 2002; Tan et al., 2008), and Dongxiang, Jiangxi province (YD4-24), the northernmost range of O. rufipogon in China (Yang et al., 2007; Xia et al., 2010), were both Stype. Although the O. rufipogon from Guangdong and Guangxi Provinces were mainly S-type and FS-type, a few F-type individuals were observed in this population. Compared to inland China, 3 O. rufipogon types were observed in Hainan Island, with FS-type $O$. rufipogon being predominant. However, notably, all of the individuals from the largest $O$. rufipogon population in Hainan Island (DA) were F-type (Table 2).

Although most $O$. rufipogon populations were composed of a single type (F, S, or FS), some populations contained 2 or 3 types. For example, 2 O. rufipogon types were present in the LHL, ZY, and PS populations and 3 O. rufipogon types were present in the DZP and TL populations. Further analysis indicated that these populations contained S and FS individuals, F and FS individuals, or S, F, and FS individuals; however, no single population was composed of only S- and F-type individuals (Table 2).

Sequence alignment revealed that $O s G I-9$ in S-type O. rufipogon contained two 255-bp fragment repeats, $O s G I-9$ in F-type $O$. rufipogon contained a single 255-bp fragment repeat, and OsGI-9 in FS-type O. rufipogon contained both the single and the double 255-bp fragment repeats (Figure 4). A small number of single base mutation sites were also observed in OsGI-9 (Figure 4).

PCR amplification of OsGI-9 in 139 O. sativa cultivars indicated the presence of Stype and F-type individuals; however, no FS-type individuals were observed. A total of 62 cultivars were S-type $(1.2 \mathrm{~kb})$ and 77 cultivars were F-type $(0.9 \mathrm{~kb})$. The $O s G I-9$ in all individuals from each cultivar were the same type (Table 3). Sequence analysis showed that $O s G I-9$ in S-type $O$. sativa had two 255-bp repeat fragments and F-type $O$. sativa had a single 255-bp repeat fragment, identical to S-type and F-type O. rufipogon individuals (Figure 4).

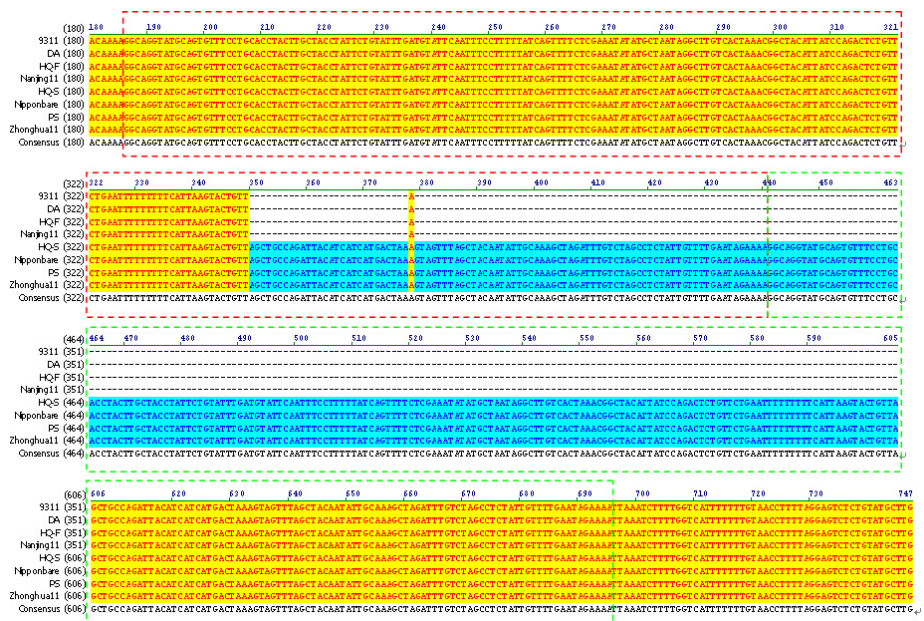

Figure 4. Sequence alignment of $O s G I-9$ in partial Oryza rufipogon and $O$. sativa. 9311 and Nanjing11 were $O$. sativa indica varieties; Nipponbare and Zhonghua11 were $O$. sativa japonica varieties; DA, PS, and HQ were $O$. rufipogon from different populations, in which HQ-F and HQ-S meant the sequences of F- and S-band in one HQ individual. Red and green dashed boxes meant two 255-bp repeated fragments. 
Y. Dong et al.

Table 3. OsGI-9 band types in 139 Oryza sativa cultivars.

\begin{tabular}{|c|c|c|}
\hline \multirow[t]{2}{*}{ Code } & \multicolumn{2}{|c|}{ Os GI-9 band type } \\
\hline & $\mathrm{S}(1.2 \mathrm{~kb})$ & $\mathrm{F}(0.9 \mathrm{~kb})$ \\
\hline Mudanjiang 19 & $\sqrt{ }$ & \\
\hline Ji 91-2605 & $\sqrt{ }$ & \\
\hline Liao 201 & $\sqrt{ }$ & \\
\hline $86 X W-17$ & $\sqrt{ }$ & \\
\hline 9011 & $\sqrt{ }$ & \\
\hline $91-13-11$ & $\sqrt{ }$ & \\
\hline Laolongxu & $\sqrt{ }$ & \\
\hline Xinong 8116 & & $\sqrt{ }$ \\
\hline Qiannong 5782 & & $\sqrt{ }$ \\
\hline Jinxi 870441 & $\sqrt{ }$ & \\
\hline Kenyu 16 & $\sqrt{ }$ & \\
\hline Zhonghua 11 & $\sqrt{ }$ & \\
\hline Zhonghua 8 & $\sqrt{ }$ & \\
\hline Zhongzuo 8604 & $\sqrt{ }$ & \\
\hline 6017 & & $\sqrt{ }$ \\
\hline Yinfang & $\sqrt{ }$ & \\
\hline Putaohuang & $\sqrt{ }$ & \\
\hline Lujing 1 & $\sqrt{ }$ & \\
\hline Zhengdao 5 & $\sqrt{ }$ & \\
\hline Xinyang 14 & & $\sqrt{ }$ \\
\hline Wanjing 1 & $\sqrt{ }$ & \\
\hline Anxuan 4 & & $\sqrt{ }$ \\
\hline Liushizao & & $\sqrt{ }$ \\
\hline Wuyujing & $\sqrt{ }$ & \\
\hline Shuangqing & $\sqrt{ }$ & \\
\hline Nanjing 11 & & $\sqrt{ }$ \\
\hline Nannong 4008 & & $\sqrt{ }$ \\
\hline 9311 & & $\sqrt{ }$ \\
\hline Shuangfeng 1 & $\sqrt{ }$ & \\
\hline Yingtoujing & $\sqrt{ }$ & \\
\hline Zheli 1 & & $\sqrt{ }$ \\
\hline Zaoxianmi & & $\sqrt{ }$ \\
\hline Zaoshu 691 & & $\sqrt{ }$ \\
\hline 3635 & & $\sqrt{ }$ \\
\hline Xiangjing 2 & $\sqrt{ }$ & \\
\hline Muguanuo & $\sqrt{ }$ & \\
\hline Xiangzaoxian & & $\sqrt{ }$ \\
\hline $86-106$ & $\sqrt{ }$ & \\
\hline Gaoyuanjing 1 & $\sqrt{ }$ & \\
\hline Meihuanuo & & $\sqrt{ }$ \\
\hline Hongmangdazu & $\sqrt{ }$ & \\
\hline Chuanmi 2 & & $\sqrt{ }$ \\
\hline Jiushizao & & $\sqrt{ }$ \\
\hline Gannongwanjing 2 & $\sqrt{ }$ & \\
\hline 4434 & & $\sqrt{ }$ \\
\hline Nante & & $\sqrt{ }$ \\
\hline Jinxibai & & $\sqrt{ }$ \\
\hline Sanbaili & & $\sqrt{ }$ \\
\hline Hongwei 1 & $\sqrt{ }$ & \\
\hline Minghui 63 & & $\sqrt{ }$ \\
\hline Dijiaowujian & & $\sqrt{ }$ \\
\hline Xixuan 4 & & $\sqrt{ }$ \\
\hline Jinxingdanuo & $\sqrt{ }$ & \\
\hline Guangluai 4 & & $\sqrt{ }$ \\
\hline GD-5S & & $\sqrt{ }$ \\
\hline Kuyexiangnuo & $\sqrt{ }$ & \\
\hline Baise 1 & & $\sqrt{ }$ \\
\hline Tainan 6 & $\sqrt{ }$ & \\
\hline Gaoxiongyu 122 & $\sqrt{ }$ & \\
\hline Jianongxianyu 31 & & $\sqrt{ }$ \\
\hline
\end{tabular}

Continued on next page 


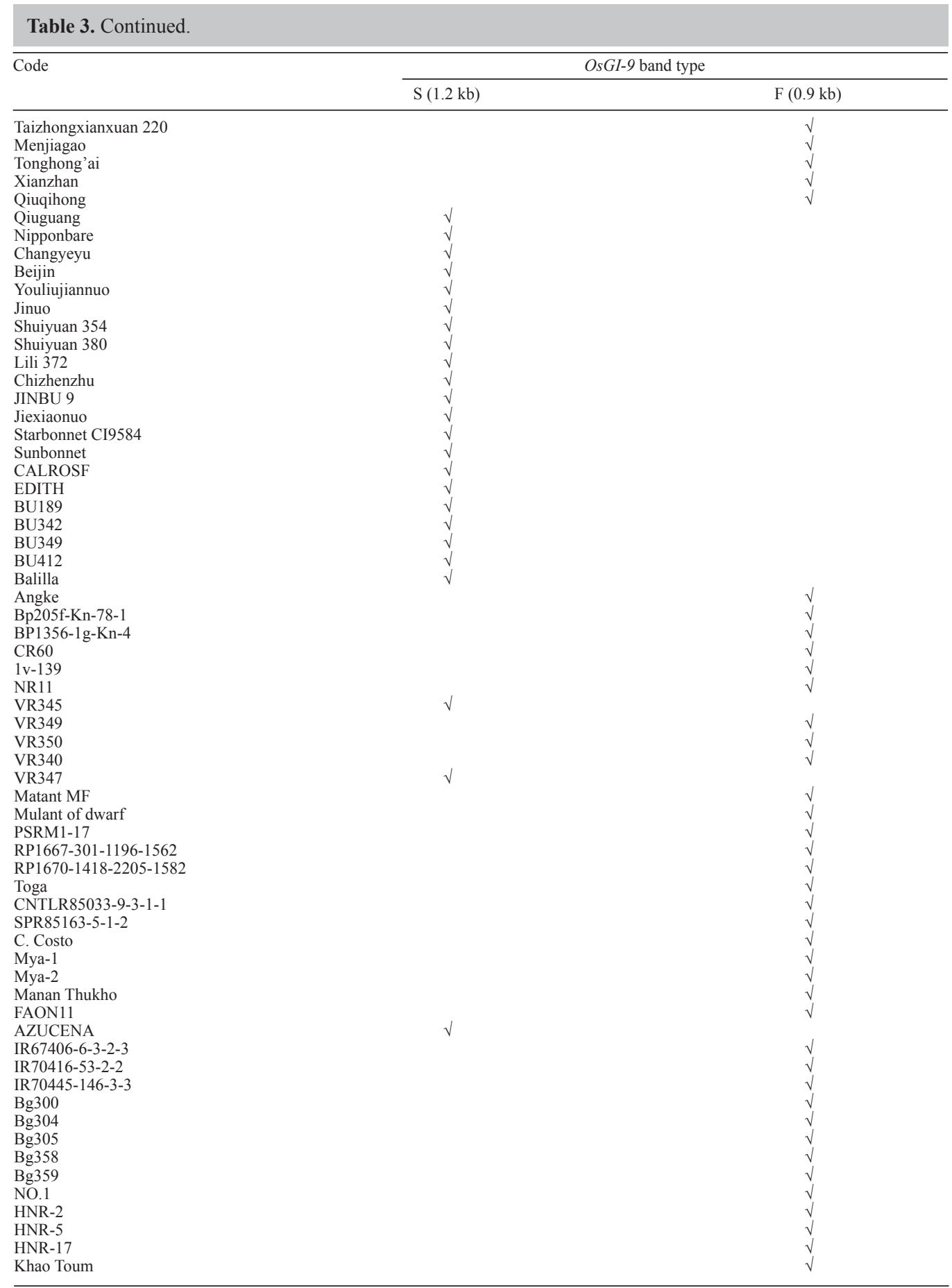

Continued on next page 


\begin{tabular}{|c|c|c|}
\hline \multirow[t]{2}{*}{$\overline{\text { Code }}$} & \multicolumn{2}{|c|}{$O s G I-9$ band type } \\
\hline & $\mathrm{S}(1.2 \mathrm{~kb})$ & $\mathrm{F}(0.9 \mathrm{~kb})$ \\
\hline Mack Kouk & $\sqrt{ }$ & \\
\hline SLK 2-21-4 & & $\sqrt{ }$ \\
\hline Mollika & & v \\
\hline NR10073-167-3-1-1 & & v \\
\hline NR10068-60-5-2 & & v \\
\hline NR10078-76-1-1 & & $\sqrt{ }$ \\
\hline Aus257 & & v \\
\hline BR061-2B-25 & & v \\
\hline BR319-1-HR28 & & $\sqrt{ }$ \\
\hline UGEY MAP & & $\sqrt{ }$ \\
\hline 80A86YR72 & $\sqrt{ }$ & \\
\hline 80A90YR73 & $\sqrt{ }$ & \\
\hline 80A97YR74 & $\sqrt{ }$ & \\
\hline 80A97YR30 & $\sqrt{ }$ & \\
\hline 71011 & $\sqrt{ }$ & \\
\hline
\end{tabular}

\section{Phylogeny of $O$. rufipogon and $O$. sativa based on OsGI-9}

The phylogenetic organism network (not haplotype) based on $O s G I-9$ indicated that all $O$. rufipogon and $O$. sativa individuals could be divided into 5 groups: 3 O. rufipogon groups (F-type, S-type, and FS-type) and 2 O. sativa groups (F-type and S-type; Figure 5).

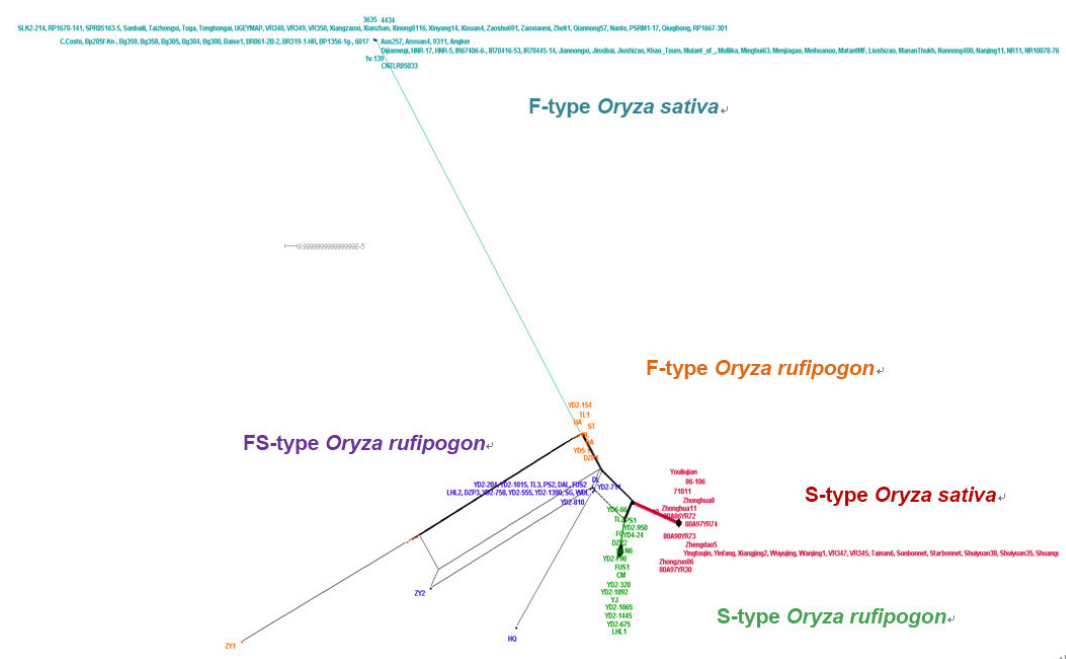

Figure 5. Phylogenetic network (NeighborNet) of the individuals from Oryza rufipogon and O. sativa based on OsGI-9.

The F-type and S-type $O$. sativa groups were located at the poles of the network, with the 3 O. rufipogon groups distributed between them (Figure 5), suggesting that the genetic distance between the $O$. rufipogon groups is less than the genetic distance between the $2 O$. sativa groups, and indicating that $O$. rufipogon is less differentiated than $O$. sativa. In the phylogenetic network, the FS-type $O$. rufipogon group was located in the middle of the $O$. rufipogon groups, and far from the $O$. sativa groups. F-type $O$. sativa was closest to F-type $O$. rufipogon, 
and S-type $O$. sativa was closest to S-type $O$. rufipogon (Figure 5). These results suggest that the $2 O$. sativa groups are more closely related than are the different $O$. rufipogon groups.

In addition, the $O$. rufipogon cultivars from HQ (FS-type), ZY1 (F-type), and ZY2 (FS-type) were located far from their respective groups (Figure 5), probably due to the presence of other OsGI-9 mutations apart from the 255-bp InDel in these individuals. Taken together, these results indicate that the $O s G I-9$ 255-bp InDel variation was retained during the domestication of $O$. rufipogon to $O$. sativa.

\section{Correlation between $O s G I-9$ and phenotype}

The $O s G I-9$ 255-bp InDel variation is common in $O$. sativa cultivars, implying that it may be significant to the adaptability of cultivars. Current studies indicate that $O s G I$ and orthologous genes play an important role in photoperiod regulation, seed dormancy, and cold tolerance (Chandler, 1992; Stewart, 2009).

The GR of $O$. sativa at $30^{\circ} \mathrm{C}$ reflects its degree of seed dormancy (Cao et al., 2001; Miura et al., 2004; Wang et al., 2009). The correlation between OsGI-9 in O. sativa and GR at $30^{\circ} \mathrm{C}$ was significant (Keudall's $r=0.279$, Spearman's $r=0.316, \mathrm{P}<0.01$; Table 4). The degree of dormancy varied in S-type $O$. sativa, while most F-type $O$. sativa were not dormant (Figure 6). The GR of S-type O. sativa was significantly lower than that of F-type $O$. sativa at $30^{\circ} \mathrm{C}(\mathrm{Z}=-3.707, \mathrm{P}<0.01$ non-parametric test; Table 5), demonstrating that the dormancy of S-type $O$. sativa is stronger than that of F-type $O$. sativa.

\begin{tabular}{|c|c|c|c|c|c|c|c|}
\hline & & \multicolumn{2}{|c|}{ GR } & \multicolumn{2}{|c|}{ GRI } & \multirow[t]{2}{*}{ GR-D } & \multirow[t]{2}{*}{ GRI-D } \\
\hline & & $30^{\circ} \mathrm{C}$ & $14^{\circ} \mathrm{C}$ & $30^{\circ} \mathrm{C}$ & $14^{\circ} \mathrm{C}$ & & \\
\hline Keudall's & OsGI-9 & $\mathrm{R}=0.279 * *$ & $\mathrm{R}=0.272 * *$ & $\mathrm{R}=0.258^{* *} *$ & $\mathrm{R}=0.254 * *$ & 0.069 & $-0.214 * *$ \\
\hline Spearman's & OsGI-9 & $\mathrm{R}=0.316^{* *}$ & $\mathrm{R}=0.311 * *$ & $\mathrm{R}=0.311 * *$ & $\mathrm{R}=0.302 * *$ & 0.080 & $-0.260^{* *}$ \\
\hline
\end{tabular}

$\mathrm{GR}=$ mean germination rate; GRI $=$ mean germination rate index; GR-D $=$ mean difference between $\mathrm{GR}$ at $14^{\circ}$ and $30^{\circ} \mathrm{C}$; GRI-D $=$ mean difference between GRI at $14^{\circ}$ and $30^{\circ} \mathrm{C} ; * * \mathrm{P}<0.01$ (two-tailed).

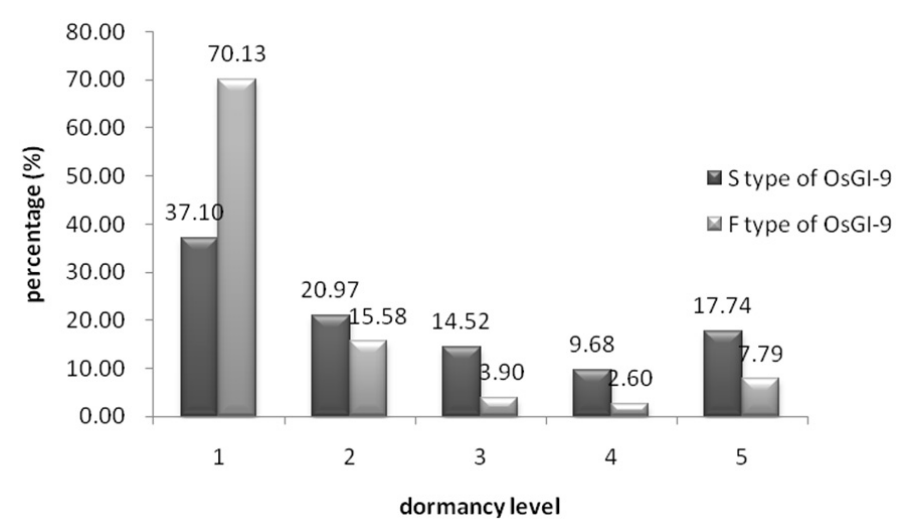

Figure 6. Frequency distribution of seed dormancy phenotype in 139 Oryza sativa cultivars. 1-5 at horizontal axis indicates GR $>80 \%, 80 \% \geq \mathrm{GR}>50 \%, 50 \% \geq \mathrm{GR}>30 \%, 30 \% \geq \mathrm{GR}>5 \%$, and $5 \% \geq \mathrm{GR}>0$, respectively. Numbers above columns account for the proportion of the total number of cultivars in the same OsGI-9 type. 
Table 5. Variation in the germination indices of 139 Oryza sativa cultivars.

\begin{tabular}{|c|c|c|c|c|c|c|}
\hline \multirow[t]{2}{*}{ OsGI-9 type } & \multirow[t]{2}{*}{ Number of cultivars } & \multicolumn{2}{|c|}{$30^{\circ} \mathrm{C}$} & \multicolumn{2}{|c|}{$14^{\circ} \mathrm{C}$} & \multirow[t]{2}{*}{ GRI-D } \\
\hline & & GR (\%) & GRI & GR (\%) & GRI & \\
\hline $\mathrm{S}$ & 62 & 59.5 & 1.9 & $\begin{array}{l}42.4 \\
685 * *\end{array}$ & 0.4 & -1.5 \\
\hline F & 77 & $80.6^{* *}$ & $2.8^{* *}$ & $68.5^{* *}$ & $0.8^{* *}$ & $-2.7 * *$ \\
\hline
\end{tabular}

For abbreviations, see legend to Table $4 . * * \mathrm{P}<0.01$ (two-tailed).

The cold tolerance of $O$. sativa was evaluated by determining the difference in GR (GR-D) or GRI (GRI-D) at $14^{\circ} \mathrm{C}$ and $30^{\circ} \mathrm{C}$. A significant correlation was observed between Os GI-9 in O. sativa and GRI-D (Keudall's $\mathrm{r}=-0.214$, Spearman's $\mathrm{r}=-0.260, \mathrm{P}<0.01$; Table 4). Although cold could significantly reduce the GR and GRI of $O$. sativa seeds, the influence of cold on GRI in S-type $O$. sativa was significantly less than in F-type $O$. sativa $(\mathrm{Z}=-3.06, \mathrm{P}$ $<0.01$; Table 5), indicating that S-type $O$. sativa have increased cold tolerance.

\section{DISCUSSION}

\section{Phylogenetic analysis of intra-individual allele heterozygotes}

Phylogeny plays an important role in reflecting evolutionary relationships among species, and for reconstructing the evolutionary process (Lessa, 1992). Due to a faster evolutionary rate, ease of amplification, near neutrality, and other evolutionary characteristics, introns have become a widely used nuclear genetic marker, and have significant potential for use in interspecific and intraspecific phylogenetic analyses (Dalebout et al., 2008). IIAHs provide a rich source of phylogenetic information and can reveal potential introgression between species or populations during the analysis of phylogenetic relationships between related species or the genetic reconstruction of populations. However, it is common practice to randomly select one haplotype from heterozygous alleles for such analyses (Göker and Grimm, 2008; Nakagome et al., 2008).

Heterozygous FS-type OsGI-9 was observed in many O. rufipogon individuals in this study. In order to determine the evolutionary relationship between heterozygous and homozygous $O$. rufipogon individuals, the genetic distance between haplotypes was converted into the genetic distance between individuals, and the individual phylogenetic network was constructed. The evolutionary relationships of heterozygous individuals cannot be analyzed using a haplotype phylogenetic tree, but can be reflected in an individual (or organism) phylogenetic network.

\section{Identification of the primitive $O$. rufipogon type}

Throughout their evolution, many types of $O$. rufipogon with rich morphological phenotypes and genetic diversity emerged in complex ecological environments (Pang and Chen, 2002; Dong et al., 2010). It is not known which types are primitive and which are derived, or how $O$. rufipogon evolved precisely. Such knowledge could help to better characterize the origin and differentiation of $O$. sativa, and promote efficient utilization of $O$. rufipogon resources.

Primitive $O$. rufipogon share few traits with $O$. sativa, having a prostrate morphology, purple leaf sheath, smaller and shorter flag, longer anther $(>5 \mathrm{~mm})$, purple and exposed stigma, red and long awn, easier seed shatter, more slender grain (length/width $>3.5$ ), black or brown hulls, and red seeds (Pang and Chen, 2002). O. rufipogon commonly grow in perennial 
swamps or rivers, and mainly reproduce asexually with a low capacity for sexual reproduction. O. rufipogon populations are generally larger; however, the morphology of individuals within an $O$. rufipogon population are identical and offspring from self-crossing rarely separate. $O$. rufipogon habitats are mostly distinct from $O$. sativa habitats, which has prevented the influence of hybridization or introgression. Compared with primitive $O$. rufipogon, many morphological changes have been observed in derived O. rufipogon, whose morphology is more or less similar to that of $O$. sativa (Pang and Chen, 2002).

In this study, individuals from the O. rufipogon YJ population in Yuanjiang, Yunnan Province, China, and YD4-24 individuals from the northern most $O$. rufipogon population in China, that are accepted to be primitive (Sun et al., 2002; Yang et al., 2007; Tan et al., 2008; Xia et al., 2010), all possessed S-type OsGI-9 (Table 2). The DA population is the largest $O$. rufipogon population in Hainan Island and grows in a perennial swamp. Individuals from this population are identical and possess the majority of primitive $O$. rufipogon morphological characteristics (Dong et al., 2010). Our results indicate that all O. rufipogon DA individuals are F-type (Table 2). On the contrary, individuals from the HQ, DL, and WDL populations do not possess primitive characteristics (Dong et al., 2010) and are all FS-type (Table 2). These results imply that F-type and S-type $O$. rufipogon are more primitive than the FS-type. The FS-type is located between F-type and S-type in the phylogenetic network (Figure 5), and FS-type individuals were always observed in populations containing both F-type and S-type individuals (Table 2), suggesting that the FS-type individuals are a hybrid of F- and S-type O. rufipogon.

O. rufipogon is commonly accepted to be the progenitor of $O$. sativa. Although $O$. sativa was domesticated from $O$. rufipogon, the process was so complex that, as yet, there is no consensus view as to how domestication occurred (Sweeney and McCouch, 2007; Sang and $\mathrm{Ge}, 2007 \mathrm{a}, \mathrm{b})$. Two diverging opinions have been proposed. One supports a multiple origin of rice domestication, suggesting that initial $O$. sativa cultivars were domesticated from divergent $O$. rufipogon populations with different sets of alleles, and introgression between independently domesticated cultivars fixed a similar set of alleles that were critical for domestication. The other opinion considers a single rice origin, suggesting that some critical domestication alleles were fixed in the initial cultivars, and then introgression between different populations of $O$. rufipogon to $O$. sativa increased cultivar diversity (Vaughan et al., 2008a,b). The phylogenetic network analysis in this study indicated that F-type $O$. sativa are closest to F-type $O$. rufipogon and that S-type $O$. sativa are closest to S-type O. rufipogon (Figure 5), supporting the multiple origin hypothesis. The presence of non-FS-type individuals in $O$. sativa cultivars is probably the result of continued domestication and breeding in different climatic conditions.

\section{OsGI 9 intron InDel}

The role of introns and intergenic sequences has received increasing attention in recent studies of gene function. Current studies indicate that introns can be divided into 3 groups. Group I and Group II introns are mainly found in organelles and bacteria and are selfcutting, although their structural features vary. Group III introns are pre-mRNA introns that are found in most eukaryotic cells (Cousineau et al., 2000). Research has indicated that intron base mutations and InDels are related to many human diseases and variation in biological characteristics (Reszka et al., 2006). 
In this study, we observed a 255-bp InDel in the ninth OsGI intron (OsGI-9). Arabidopsis GI, the $O s G I$ rice homolog, which regulates photoperiod-mediated flowering and the circadian clock, can affect fruit setting and cold tolerance (Fowler et al., 1999; Brock et al., 2007). In this study, OsGI-9 variation in $O$. sativa was associated with seed dormancy and cold tolerance (Tables 4 and 5), but was not associated with photoperiod (data not shown). Most Stype $O$. sativa cultivars had strong seed dormancy and high cold tolerance and most F-type $O$. sativa cultivars had poor seed dormancy and low cold tolerance (Figures 1 and 6; Tables 3 and 5). Therefore, we speculate that the 255-bp OsGI-9 InDel is correlated with seed dormancy or cold tolerance in $O$. sativa.

\section{ACKNOWLEDGMENTS}

We thank the Crop Science Research Institute in CAAS and the Jiangxi Academy of Agricultural Sciences for providing partial samples. Research supported by grants from the National Special Program of Transgenic Research (\#2011ZX08011-001 and \#2013ZX08011001) and the National Nonprofit Program of Basic Research (\#1610392011010).

\section{REFERENCES}

Brock MT, Tiffin P and Weinig C (2007). Sequence diversity and haplotype associations with phenotypic responses to crowding: GIGANTEA affects fruit set in Arabidopsis thaliana. Mol. Ecol. 16: 3050-3062.

Bryant D and Moulton V (2004). Neighbor-net: an agglomerative method for the construction of phylogenetic networks. Mol. Biol. Evol. 21: 255-265.

Cao YJ, Jiang L, Luo L, Zhai H, et al. (2001). A study on seed dormancy in rice (Oryza sativa L.). J. Nanjing Agric. Univ. 24: $1-5$.

Chandler RF (1992). An Adventure in Applied Science: A History of the International Rice Research Institute. IRRI Press, Philippines.

Cousineau B, Lawrence S, Smith D and Belfort M (2000). Retrotransposition of a bacterial group II intron. Nature 404: 1018-1021.

Creer S (2007). Choosing and using introns in molecular phylogenetics. Evol. Bioinform. Online 3: 99-108.

Dalebout ML, Steel D and Baker CS (2008). Phylogeny of the beaked whale genus Mesoplodon (Ziphiidae: Cetacea) revealed by nuclear introns: implications for the evolution of male tusks. Syst. Biol. 57: 857-875.

Dong YB, Pei XW, Yuan QH, Wu HJ, et al. (2010). Ecological, morphological and genetic diversity in Oryza rufipogon Griff. (Poaceae) from Hainan Island, China. Genet. Resour. Crop Evol. 57: 915-926.

Doyle JJ, Kanazin V and Shoemaker RC (1996). Phylogenetic utility of histone H3 intron sequences in the perennial relatives of soybean (Glycine: Leguminosae). Mol. Phylogenet. Evol. 6: 438-447.

Felsenstein J (2008). PHYLIP (Phylogeny Interference Package), Ver. 3.68. Department of Genome Sciences and Department of Biology. University of Washington, Seattle.

Fowler S, Lee K, Onouchi H, Samach A, et al. (1999). GIGANTEA: a circadian clock-controlled gene that regulates photoperiodic flowering in Arabidopsis and encodes a protein with several possible membrane-spanning domains. EMBO J. 18: 4679-4688.

Göker M and Grimm GW (2008). General functions to transform associate data to host data, and their use in phylogenetic inference from sequences with intra-individual variability. BMC Evol. Biol. 8: 86.

Huson DH and Bryant D (2006). Application of phylogenetic networks in evolutionary studies. Mol. Biol. Evol. 23: 254-267.

Larkin MA, Blackshields G, Brown NP, Chenna R, et al. (2007). Clustal W and Clustal X version 2.0. Bioinformatics 23: 2947-2948.

Lessa EP (1992). Rapid surveying of DNA sequence variation in natural populations. Mol. Biol. Evol. 9: 323-330.

Lu BR, Zheng KL, Qian HR and Zhuang JY (2002). Genetic differentiation of wild relatives of rice as assessed by RFLP analysis. Theor. Appl. Genet. 106: 101-106.

Miura K, Lin SY, Araki H, Nagamine T, et al. (2004). Genetical studies on germination of seed and seedling establishment for breeding of improved rice varieties suitable for direct seeding culture. Jpn. Agr. Res. Q 38: 1-5. 
Nakagome S, Pecon-Slattery J and Masuda R (2008). Unequal rates of $\mathrm{Y}$ chromosome gene divergence during speciation of the family Ursidae. Mol. Biol. Evol. 25: 1344-1356.

Pang HH and Chen CB (2002). Chinese Wild Rice Resource. Guangxi Science and Technology Press, Guangxi.

Rakshit S, Rakshit A, Matsumura H, Takahashi Y, et al. (2007). Large-scale DNA polymorphism study of Oryza sativa and O. rufipogon reveals the origin and divergence of Asian rice. Theor. Appl. Genet. 114: 731-743.

Ren F, Lu BR, Li S, Huang J, et al. (2003). A comparative study of genetic relationships among the AA-genome Oryza species using RAPD and SSR markers. Theor. Appl. Genet. 108: 113-120.

Reszka E, Wasowicz W and Gromadzinska J (2006). Genetic polymorphism of xenobiotic metabolising enzymes, diet and cancer susceptibility. Br. J. Nutr. 96: 609-619.

Sang T (2002). Utility of low-copy nuclear gene sequences in plant phylogenetics. Crit. Rev. Biochem. Mol. Biol. 37 : 121-147.

Sang T and Ge S (2007a). Genetics and phylogenetics of rice domestication. Curr. Opin. Genet. Dev. 17: 533-538.

Sang T and Ge S (2007b). The puzzle of rice domestication. J. Integr. Plant Biol. 49: 760-768.

Slade RW, Moritz C and Heideman A (1994). Multiple nuclear-gene phylogenies: application to pinnipeds and comparison with a mitochondrial DNA gene phylogeny. Mol. Biol. Evol. 11: 341-356.

Stewart CN (2009). Weedy and Invasive Plant Genomics. Blackwell Publishing, USA.

Sun CQ, Wang XK, Li ZC, Yoshimura A, et al. (2001). Comparison of the genetic diversity of common wild rice (Oryza rufipogon Griff.) and cultivated rice (O. sativa L.) using RFLP markers. Theor. Appl. Genet. 102: 157-162.

Sun Q, Wang K, Yoshimura A and Doi K (2002). Genetic differentiation for nuclear, mitochondrial and chloroplast genomes in common wild rice (Oryza rufipogon Griff.) and cultivated rice (Oryza sativa L.). Theor. Appl. Genet. 104: $1335-1345$.

Sweeney M and McCouch S (2007). The complex history of the domestication of rice. Ann. Bot. 100: 951-957.

Tan L, Zhang P, Liu F, Wang G, et al. (2008). Quantitative trait loci underlying domestication- and yield-related traits in an Oryza sativa x Oryza rufipogon advanced backcross population. Genome 51: 692-704.

Vaughan DA, Lu BR and Tomooka N (2008a). The evolving story of rice evolution. Plant Sci. 174: 394-408.

Vaughan DA, Lu BR and Tomooka N (2008b). Was Asian rice (Oryza sativa) domesticated more than once? Rice 1: $16-24$.

Wang ZF, Wang JF, Wang FH, Bao YM, et al. (2009). Genetic control of germination ability under cold stress in rice. Rice Sci. 16: 173-180.

Xia RX, Xiao N, Hong YH, Zhang C, et al. (2010). QTLs mapping for cold tolerance at seedling stage in Dongxiang wild rice (Oryza rufipogon Griff.). Sci. Agric. Sin. 43: 443-451.

Xie ZW, Ge S and Hong DY (1999). Preparation of DNA from silica gel dried mini-amount of leaves of Oryza rufipogon for RAPD study and total DNA bank construction. Acta Bot. Sin. 41: 807-812.

Xie ZW, Lu YQ, Ge S, Hong DY, et al. (2001). Clonality in wild rice (Oryza rufipogon, Poaceae) and its implications for conservation management. Am. J. Bot. 88: 1058-1064.

Yang QW, Yu LQ, Zhang WX, Shi J, et al. (2007). The genetic differentiation of Dongxiang wild rice (Oryza rufipogon Griff.) and its implications for in-situ conservation. Sci. Agric. Sin. 40: 1085-1093. 